\title{
Estado del arte de la investigación "inclusión del componente pedagógico, desde lo crítico, en los principios de la ética de mínimos y máximos de Adela Cortina en la categoría ciudadanía"
}

\author{
Álvaro Hernández Acevedo ${ }^{1}$
}

Recibido: 14-03-2017

Aceptado: 07-04-2017

\section{RESUMEN}

Este trabajo muestra una aproximación al estado del arte de la investigación acerca de la formación en ciudadanía, partiendo de la inclusión del componente pedagógico, desde lo crítico, a las distintas formas políticas educativas de ser y generar ciudadanos de y para el mundo. Se parte de la comprensión de las múltiples acepciones que se han venido estableciendo acerca de ciudadanía, su relación con las dimensiones humanas, especialmente lo político, económico, social y cultural. En este contexto, se plantea la función de la pedagogía en el cómo, qué y para qué integrar el aula, las experiencias vitales y la teoría que incluyen el ser ciudadano, además que las relaciones que se establecen para lograr una mentalidad ciudadana, crítica y emancipadora de los sistemas hegemónicos.

Palabras clave: ciudadanía, modos de ciudadanía, educación y pedagogía para la ciudadanía, inclusión, pedagogía crítica. 


\title{
State of the art of the research "inclusion of the pedagogical component, from the criticism in the principles of minimum and maximum ethics by Adela Cortina in the citizenship category"
}

\begin{abstract}
This paper aims to show an approximation to the state of the art of research on training in citizenship, based on the inclusion of the educational component, from the critical thought, the various educational policies and generate ways of being citizens and the world. It is part of the understanding of the multiple meanings that have been setting about citizenship, their relationship to human dimensions, especially the political, economic, social and cultural. In this context, the role of pedagogy in arises how, why and what to integrate the classroom, life experiences and theory including being a citizen, in addition to the relationships established to achieve a civic -minded, critical and emancipation of hegemonic systems.
\end{abstract}

Keywords: Citizenship, ways of citizenship education and education for citizenship, inclusion, critical pedagogy.

"Ojalá que de niños nos enseñaran para luchar por ideales y no por calificaciones. Así de adultos, trabajaríamos por sueños y no por quincenas" Anónimo

\section{Introducción}

Este trabajo intenta mostrar las condiciones de las investigaciones acerca de la relación que se puede establecer entre pedagogía crítica, formación en ciudadanía y los aportes teóricos de filósofos que se han preocupado por la ciudadanía como un estilo de vida, y como 
una categoría esencial, que incluye dimensiones como lo político, lo educativo, lo social y lo cultural mediadas por lo ético. En este sentido, en el marco de la investigación del autor, sirve como marco de comprensión para la inclusión de lo pedagógico, desde la perspectiva crítica, guiado por Peter McLaren y Henry A. Giroux, a los principios de una ética mínima establecidos por Adela Cortina aplicados a la formación en ciudadanía.

La formación en ciudadanía es una preocupación sentida por todas las instituciones educativas, estatales y de la sociedad civil, ya que se viene comprendiendo que la participación activa, la deliberación entre los individuos, el disenso y el consenso, el compromiso por las decisiones consensuadas, el respeto absoluto por el otro, la defensa, promoción y protección de los derechos, en aras de lograr estrategias, mecanismos y proyectos para lograr un entorno de justicia social y desarrollo sostenible, son dones y tareas para lograr una sociedad democrática, ciudadana, cuyos imaginarios están marcados entre lo global y lo local, de tal forma que los miembros asumen un estilo de vida donde hacen una lectura crítica de su realidad, se hallan "emancipados" de los sistemas hegemónicos y totalitarios de la actualidad.

En 1990 en Barcelona se realizó el primer Congreso Internacional de Ciudades Educadoras, donde se estableció que una ciudad era educadora en sí misma, ya que en ella se desenvuelven todos los criterios, principios y valores que llevan a la consolidación de una democracia donde la pedagogía, la información, la participación política y las relaciones personales son esenciales para la construcción de ciudadanía. Así lo afirma Jaume Trilla Bermett:

El concepto de Ciudad Educadora acoge e interrelaciona procesos educativos formales, no formales e informales. La ciudad educativa es un entramado de instituciones y lugares educativos. Los nudos más estables y obvios de esta trama están constituidos por las instituciones formales de educación (escuelas, universidades, etc.). Pero coexisten con ellas, por un lado, todo el conjunto de intervenciones educativas no formales (organizadas a partir de objetivos explícitos de formación o enseñanza, pero fuera del sistema de la enseñanza reglada: educación en el tiempo libre, auto-escuelas, etc.), y, por otro lado, el difuso y penetrante conjunto de vivencias educativos informales (espectáculos, publicidad, relaciones de amistad, etc., etc.). Quizás el medio urbano sea precisamente el mejor 
ejemplo de las constantes interacciones entre estos modos de educación (Citado por Cabezudo, 2006).

De ahí que la relación entre pedagogía, ciudadanía y sociedad son categorías básicas para consolidar una verdadera, plena y justa democracia. Por esta razón, es vital realizar un recorrido acerca de las investigaciones que se han desarrollado en el contexto de la pedagogía crítica para la formación en ciudadanía, como es el caso de la pedagogía de Educar en la Calle con jóvenes del Distrito de Aguablanca en Cali (2010), cuya pregunta problémica fue ¿Qué construcción de sentidos sobre ciudadanía desarrollan jóvenes hombres y mujeres del Distrito de Aguablanca de Cali, que han participado en un proceso de Educar en la Calle y cómo éste ha influido en su constitución como sujetos sociales y políticos? Esta investigación llegó a concluir que en la formación de ciudadanos se entrelazan posibilidades, sensibilidades, diferencias, narrativas, vitalidades, exclusiones e inclusiones en las experiencias, lo cual ha generado encrucijadas en la mentalidad juvenil, aunque responde a lo que se podría entender, en términos de Kymlicka, como una ciudadanía diferenciada, esto es la "adopción de derechos poliétnicos, de representación o de autogobierno específicos en función del grupo" (1996, p. 241). Además, la pedagogía (Valencia \& Giraldo, 2005) es esencial para la integración del aula con la vida cotidiana para ser proyectivos, incluyentes y comprometidos para lograr una sociedad democrática y ciudadana.

En el contexto inmediato de Tunja, haciendo un mapeo se encontró que ya en 1997 se iniciaron proyectos de formación de parte de la sociedad civil, como tal es el caso de la corporación Amor a Tunja, conformada alrededor de 90 mujeres que presentaron un proyecto de Fortalecimiento de la Cultura Ciudadana, en el que desarrollaron diferentes temáticas como Ornato y Embellecimiento, Ecología, Educación del Tránsito, Arte y Turismo, Escuela de Formación de Líderes, Redacción y Publicidad, Identidad Tunjana, Valores Cívicos y Sociales, y Estrategias Educativas, evidenciando la visión multifacética de la comprensión de la ciudadanía, evidenciando que la ciudadanía como un estilo de vida sólo es posible en la interacción de todas las dimensiones del ser humano en relación con el otro.

Este trabajo tuvo como objetivo "crear en los ciudadanos tunjanos una verdadera conciencia de amor y civismo por la ciudad, propiciando ambientes y metodologías educativas para trabajar en acción comunitaria 
en proyectos, planes, programas y actividades que estimulen el desarrollo, progreso y adelanto de Tunja" (1997). Iniciativas como estas se realizaron a partir de la elaboración, análisis y divulgación de libros, folletos, mapas y cartillas cívicas y turísticas, programas de sensibilización dentro de la ciudadanía, elaboración de publicidad educativa en torno a la ciudad, conformación de una escuela de líderes cívicos con espacios concretos para ejercer el liderazgo, y la realización de foros, conferencias, talleres y encuentros para fortalecer la cultura ciudadana, la cual, como lo anota Camps, sigue convirtiéndose en tarea del pensamiento práctico de la filosofía política y especialmente de la mentalidad liberal que "no ha sabido ir más allá de una concepción excesivamente jurídica y formal de ciudadanía" (2010, p. 9).

Además, era necesario mejorar los parques existentes en la ciudad y crear brigadas de cultura en las juntas de acción comunal. Estas acciones nacieron luego de la reflexión conjunta entre los diferentes centros de educación, sociedad civil y la participación del Estado, evidenciando lo que se ha venido demostrando hasta el momento, la necesidad de la intervención de las diferentes dimensiones humanas, aunque se denota la falta de la inclusión de propuestas venidas de la pedagogía que interiorizan la vida cotidiana con el aula, a lo que pretende desarrollar este trabajo de investigación, pretendiendo que el aprendizaje construido en el aula sea el eje articulador de una mentalidad ciudadana crítica y emancipada de los estereotipos culturales y sistemas hegemónicos que forman ciudadanos negados en sus derechos básicos.

Para ello, es necesaria la vinculación de todos los agentes tanto del acto educativo como de la sociedad en general de tal manera que puedan hablar, ser escuchados, generar relatos venidos de la memoria y la narrativa, como es el caso de las políticas educativas expresadas en el diseño curricular de construcción de ciudadanía de la región de La Plata en Argentina (2008).

\section{Ciudadanías y pedagogía}

Al respecto, Wilson Muñoz en su trabajo "La ciudadanía pedagógica, una reflexión de ciudadanía democrática desde el proceso educativo" (2005) demostró que, a lo largo de la historia de Chile, se han formado ciudadanos negados en sus derechos y en su capacidad de participación 
activa. Explicó la necesidad de concebir al maestro como un sujeto político (Freire) que forma ciudadanos; además que, si niega la participación activa de sus estudiantes y no genera espacios de formación ciudadana, niega su esencia como pedagogo. Concluye, por tanto, que la ciudadanía neoliberal ha generado la exclusión de la mayoría de los individuos, convirtiéndolos en ciudadanos de segunda categoría, y que Chile ha asumido una pedagogía social que tiene como objetivo ser una "disciplina que tiene por objetivo el conjunto de acciones educativas no formales orientadas al desarrollo de la sociabilidad de los sujetos (ciudadanía y democracia participativa) y colectivos que viven algún tipo de situación conflictiva en su entorno social (Ascensio, 1999 citado por Muñoz, 2005, p. 77), aunque esta política educativa ha generado ciudadanos imaginarios, en palabras de Saavedra (2002).

Así, uno de los retos más importantes de la educación y la pedagogía es establecer políticas, mecanismos y estrategias para unir las brechas establecidas entre las formas de educar a nuestros estudiantes con los nuevos retos que se presentan para desarrollar una sociedad de ciudadanos de primera en una sociedad verdaderamente democrática. En este sentido, se entiende lo que Fajardo (2016) plantea de generar en el aula espacios y ambientes democráticos para analizar la sociedad y sus contextos desde la reflexión filosófica política, formándolos en capacidades y habilidades sociales, valores cívicos y convivencia ciudadana desde la justicia social, la verdad, la participación, el respeto por el otro y la valoración de la propia realidad individual. De ahí que la pedagogía, bajo estos principios, forma sujetos ciudadanos que respetan los derechos del otro y de lo otro.

En este estado de la cuestión se plantearon algunas categorías que indicaran el proceso investigativo acerca de esta temática, entre las cuales se establecieron pedagogía crítica, ciudadanía, formación ciudadana, Adela Cortina, Peter McLaren, Henry A. Giroux, las cuales arrojaron una serie de investigaciones que aludían tangencialmente a la aplicación del componente pedagógico desde lo crítico a los procesos formativos de ciudadanía desde la perspectiva de Adela Cortina. Se evidenció una profusión de artículos, tesis de maestrías y doctorados acerca de ciudadanía vinculados a procesos políticos, la misma conceptualización a lo largo de la historia, las relaciones entre formación, instituciones educativas y currículo en pro de esta categoría, más la inclusión de lo pedagógico crítico, con una lectura de Peter McLaren o de Giroux a la propuesta de Adela Cortina, no se arrojaron ningún resultado, lo que prioriza la necesidad de esta investigación. 
Tal es el caso de Cárcamo-Vásquez (2015), quién en su trabajo "Ciudadanía y formación ciudadana", tuvo como objetivo fundamental la comprensión del modo en que influye la formación inicial docente en la configuración de las representaciones sociales que posee el estudiantado que se forma para ser maestro sobre ciudadanía y la formación ciudadana. En su trabajo, realizado mediante un enfoque etnográfico de observación participante, se interesaron por un análisis de las prácticas y experiencias vividas por los futuros maestros que debían abordar los temas de ciudadanía y formación ciudadana; desarrollaron la revisión documental, relatos de docentes, diarios de campo y entrevistas en profundidad para identificar la forma en que se desarrollaban las clases, tomando en consideración elementos como el discurso docente, las metodologías utilizadas, la disposición espacial del estudiantado en ella, así como la participación y el discurso de este en torno a las temáticas abordadas en la asignatura (p. 2).

Los autores establecieron que la formación ciudadana y su manera de concebirla están mediadas por una visión de educación centrada en aspectos técnicos pedagógicos. Así la didáctica es fundamental para reproducir el orden social, lo que confirma que la forma (pedagogía) en que se genera el discurso de la ciudadanía en función de la sociedad, enmarca la tipología de educación que se pretende brindar a la sociedad (discurso político de la educación), sustentando así los sistemas hegemónicos de los que es necesario lograr una emancipación, ya que es vital reconocer la importancia de desnacionalizar el concepto ciudadanía, lo que vincula más dimensiones humanas que van desde la objetiva (existencia de derechos) hasta las subjetivas (acercamiento al ejercicio de derechos), en función de integrar en la escuela la formación de ciudadanos (p. 2). Para los autores y la esencia de la pedagogía crítica es imprescindible la formación ciudadana tanto de maestros como de estudiantes, por lo que es necesario vincular la vida cotidiana en el salón de clases (Hernández 2015, 2016, p.2), para lograr las metas de la formación de ciudadanos:

aportar a construir la paz, fomentar la participación
democrática...empoderar a los estudiantes para participar
democráticamente en la sociedad y desarrollar las
competencias necesarias para el uso constructivo de esta
participación (Fajardo, 2016, p.93).

En su trabajo, Cárcamo-Vásquez, desarrolla la teoría de las representaciones sociales, basándose en Moscovici (1979), para la 
formación en ciudadanía en los futuros maestros, constata que la formación en ciudadanía es vital para lograr una actitud reflexiva y crítica en los estudiantes, tanto de los que tendrán en un futuro como de ellos mismos, de tal forma que sea una realidad esta competencia, y que los valores rescatados para una ciudadanía están la tolerancia, solidaridad, fraternidad, caridad y la compasión.

Ahora bien, ha sido una constante la importancia de plantear algunos referentes conceptuales acerca de ciudadanía, pues a partir de esta postura surgen las múltiples formas pedagógicas y educativas para formar ciudadanos. Este fue el objetivo que Giraldo se planteó en su trabajo "Ciudadanía: aprendizaje de una forma de vida" (2015), quien mediante una revisión documental sobre los enfoques sobre ciudadanía en cuanto evolución, transformación e implicación, y contraste con los debates y cuestionamientos que se hacen en la actualidad, realizó un análisis histórico sobre modelos y enfoques clásicos y modernos acerca de la ciudadanía para esclarecer su origen, desarrollo, sentido profundo y vigencia.

Así, comprendió que la concepción de ciudadanía contemporánea difiere de la clásica, tanto en lo referente a los derechos como en la manifestación de desigualdad, crisis social y emergencia de otros intereses (económicos), que gobiernan las sociedades modernas. Además, el ciudadano es un ser político con una dimensión social y moral, porque la construcción de ciudadanía no es el aprendizaje de normas, sino la realización efectiva de una forma de vida y de convivencia entre los seres humanos en sociedad. Giraldo concluye que la ciudadanía es un concepto en constante construcción evolutiva que obedece a los cambios e intereses histórico-sociales (2015, p. 14), y la apuesta radica en hacerlo relevante y pertinente para un mundo fragmentado y globalizado, y más aún, que lleve a la promoción y fortalecimiento para la democracia, incluyendo categorías como poder, sistemas económicos y perspectivas culturales. Así mismo lo plantea Ramírez (2012), en la necesidad de incluir a todos los agentes en condición igualitaria para eliminar toda exclusión de los menos favorecidos, y se favorece la búsqueda de sujetos responsables e identidades conscientes de su posición en el mundo para actuar en el entorno inmediato humano.

En la revisión trae a colación definiciones contemporáneas como "la ciudadanía supone y representa ante todo la plena dotación de derechos que caracteriza al ciudadano en las sociedades democráticas 
contemporáneas (Arango, 2006, p. 1). Esta afirmación hace énfasis en realización efectiva de los derechos y no solamente en la promulgación legal. A lo que faltaría, además, el componente pedagógico al discurso formativo de la ciudadanía en todas las dimensiones, lo cual tiene el texto relevancia en esta comprensión de la importancia de la adhesión y el reconocimiento del ciudadano como tal y de la sociedad a éste (Cortina, 1998, p. 25).

Giraldo concluye, además, que es necesaria la formación en ciudadanía para la vivencia plena de los derechos a los que tiene por su misma condición de vivir en un estado democrático, es decir, es una condición y es una tarea de cada individuo, del que la educación adquiere un papel preponderante y la pedagogía una labor incesante de generar mentalidades ciudadanas, en donde la identidad como tal es conditio sine qua non para vivir en un ambiente democrático, puesto que, a la hora de comprender y formar en ciudadanía, se deben generar procesos pedagógicos de identidad al territorio, a su historia, y a su sociedad en todas sus dimensiones para exigir el disfrute de los derechos a los que tiene lugar, lo cual permitirá concluir que la ciudadanía no es el aprendizaje mecánico de unas normas (jurídicas, legales y políticas), sino la realización efectiva de una forma de vida y de convivencia entre los seres humanos en sociedad bajo principios de participación y compromiso.

\section{Ciudadanía y pedagogías emancipatorias}

En función de estos dos principios, Streck y Zazini (2013), en su trabajo "Colonialidade e insurgência: contribuições para uma pedagogía latinoamericana", al discutir la pedagogía latinoamericana y la producción de conocimiento, en una perspectiva de superación de colonialidad pedagógica, analizaron las características de las prácticas pedagógicas enmarcadas en la visión emancipatoria en América Latina, influenciadas por Paulo Freire, recuperando los elementos de memoria en torno a la idea de colonialidad, la superación de la misma y la educación, de tal forma que se pueda comprender la necesidad de generar procesos de resistencia ante la colonialidad y enmarcar el valor de la insurgencia en la pedagogía. Es así que concluyeran que es necesario un diálogo horizontal entre conocimientos para buscar metodologías propias, establecer un espacio de resistencia o movimientos de lugares y tiempos diversos, y la importancia de buscar la latinidad negada o aprender en las fronteras. 
Para ello es esencial el recurso de la búsqueda de la memoria pedagógica latinoamericana como un proceso abierto para superar la colonialidad desde las insurgencias pedagógicas, lo cual significa anticipar y ensayar las posibilidades endógenas y auténticas, puesto que no se puede seguir estableciendo estándares de poder eurocéntrico y capitalista que impone identidades ajenas al sujeto latinoamericano.

Los autores traen a colación el movimiento de trabajadores rurales Sem Terra (MST) y el Sistema educativo rebelde y autónomo Zapatista (SERAZ), como ejemplos de la comprensión del sujeto político que piensa el sentido del poder en contra de los sistemas totalitarios capitalistas. De ahí la organización de cooperativas y bases de apoyo que crean conciencia social y de construcción de identidad sin mundos preestablecidos (2013, p. 40). En relación con la función de la pedagogía, definen como fundamental la relación dinámica entre lenguaje y realidad en el ejercicio de comprensión del contexto y la práctica, de tal forma que se favorece la variedad de las complejidades y manifestaciones sobre la aprensión de la realidad.

En una pedagogía crítica, la realidad es una creación histórica y cultural, y por ello puede ser transformada por las representaciones ideológicas dadas entre el ser y las condiciones de vida de esa realidad. Esta visión permite la inclusión de nuevas perspectivas y el compromiso de cada estudiante para que hagan una lectura crítica de la realidad y de los mitos de los discursos dominantes que oprimen y excluyen, y así lograr la autenticidad cultural, étnica y política.

La resistencia, por tanto, ante el colonialismo, inicia con un conocimiento desde el entorno propio con una mirada plural e incluyente hacia una corresponsabilidad de construcción de consensos, elemento esencial para desarrollar una pedagogía liberadora, decolonial donde la memoria juega ese papel preponderante, ya que vincula el pasado que proyecta un futuro para realizarlo en el presente. Ahora bien, los autores, siguiendo a Freire, destacan que es necesaria una educación para la decisión, para la política y así, para la transformación y la emergencia de nuevas ideas nacidas de la insurgencia (2013, p. 42), pues sólo así será libertadora en las dinámicas presentes, en los diálogos y en las relaciones horizontales, las cuales generan territorios y lugares de resistencia, en cuanto que establecen relaciones humanas desde lo cotidiano, con la naturaleza y sus recursos que luchan contra los sistemas totalizantes, y así buscar la latinidad y el aprendizaje desde 
las fronteras, volviendo a las culturas ancestrales que podrán liberar el pensamiento latinoamericano y definir una ciudadanía de acuerdo con los parámetros endógenos de una región que posee una riqueza merecedora de una seria ecología de saberes, siempre y cuando se enseñe desde la mirada glocal, es decir, a partir de un pensamiento global y una acción local.

Ahora bien, en el proceso de formación de ciudadanía y de sus distintas concepciones, y las maneras de cómo se debe enseñar, se han venido desarrollando el tema de las competencias ciudadanas, apuntando, desde el Ministerio de Educación Nacional, a que el ciudadano sea partícipe, con actitud constructiva y activa en la sociedad, ya que es claro que la ciudadanía va más allá del cumplimiento y ejercicio de los derechos y deberes incluyendo así la participación activa en la comunidad, el conocimiento de su entorno social y político, su reflexión crítica sobre los problemas de su ambiente vital, y generador de soluciones posibles a estos mismos problemas (MEN, 2006, p. 149; 2013, p. 2).

En estos documentos oficiales no se hace eco sobre la pregunta por la pedagogía que respondería a los intereses de una sociedad o, más aún, del Estado ni mucho menos en el tipo de ciudadano que se busca para la nación colombiana. por esto Montoya afirma que:
Un país como Colombia, lastrado por altísimos niveles de corrupción, de violencia y de desigualdad social, no debe plantearse como meta únicamente la formación de profesionales con calidad técnica; es fundamental el papel que pueden desempeñar las ciencias humanas y sociales en la construcción de una democracia más justa e incluyente (2011, p.48)

En este marco de comprensión, Magendzo (2007), analizó la relación y sentido del papel de la educación para formar ciudadanos, mediante una revisión documental, concluyendo que dependiendo del valor y concepción que le demos a la democracia, así será nuestra forma de comportamiento ante las distintas formas de ordenamiento social y moral. Entonces, la ciudadanía es una forma de mirar el mundo y lleva un implícito moral en la decisión conceptual ante la democracia (p. 71). El autor, defiende que la democracia deliberativa es un medio que fortalece la ciudadanía, y en la escuela es vital incluir un currículo que llevaría a la 
deliberación, desarrollando una pedagogía deliberativa que se nutre de la pedagogía crítica², de la alteridad y de la problematizadora. En este particular, Cortina establece que sigue siendo necesaria la formación en las virtudes (1993, p. 213) y éstas para la democracia, porque sólo en la consecución de hábitos y habilidades para reconocer al otro llevará un autoreconocimiento que posibilita la experiencia en proyecto de una verdadera autonomía que construye comunidades dialógicas comprometidas con un proyecto común.

Este trabajo es de gran importancia para nuestra investigación, puesto que resalta la importancia de la relación que surge al momento de pensar una sociedad democrática basada en actitudes de ciudadanía, en la que verdaderamente el individuo se asuma como ciudadano, sea aceptado por la comunidad, genere disensos y lleguen, en conjunto a consensos con sus correspondientes compromisos de acción pertinente y relevante.

En este sentido, la democracia deliberativa tiene como ejes centrales la autonomía de la persona, la inviolabilidad de la persona y la dignidad de la persona para generar una discusión razonada, respetuosa y abierta al otro, con lo cual se puede llegar a acciones concretas, comprometidas del colectivo. Entonces, siguiendo a Cortina, la autonomía se considera como una categoría esencial como fundamento de deberes universalmente exigibles, y de ahí ser universalmente respetadas (2000, p. 23). En ella se incluyen los principios intersubjetivos, los ideales personales sin limitar el bienestar de otros; de ahí que sea tan importante comprender que en la democracia deliberativa es esencial la convivencia de intereses distintos en un espacio común, donde lo primordial son las razones que sustentan los intereses y no éstos en sí mismos.

En esta misma línea el trabajo cualitativo y cuantitativo de Bedoya Santana (2003) denominado "Hacia una pedagogía para la ciudadanía", en la que estudiaron los diferentes tópicos relacionados con la ciudadanía y la democracia en los estudiantes de la Universidad Distrital Francisco

2. "La pedagogía crítica comprende el mundo de lo educativo como una empresa que incide en lo político, cultural y social, lo que ha generado que olvide su propia y más genuina misión; a lo que responde con el llamado de atención ante el verdadero papel de la escuela en la sociedad, de ahí que favorezca la reestructuración de los modelos, los procesos educativos y el currículo en todas sus manifestaciones, pues éste debe vincular lo teórico con la práctica, con la vida cotidiana, con los intereses, deseos y anhelos de los agentes del asunto pedagógico. Cuando se hace alusión al cuerpo, se llama la atención a la importancia de lo corporal, es decir, a que actualmente nos hallamos ante una resistencia para aprender porque el estudiante es asumido como un receptor pasivo, y su corporalidad no tiene ninguna participación en el acto pedagógico". (Hernández, 2016, p. 1) 
José de Caldas. Las autoras determinaron los conceptos fundamentales de su investigación y los analizaron mediante entrevistas, encuestas y observación participante, los cuales fueron identidad y pertenencia, derechos políticos y sociales, participación, libertad y dignidad, desarrollo humano y responsabilidad humana y colectiva. Entre sus conclusiones establecieron que existe una encrucijada frente a la construcción de ciudadanía en medio de los profesionales en formación, puesto que se evidencia una serie de imaginarios, concepciones, opiniones, actitudes y valores alrededor de la democracia y ciudadanía, lo que lleva a establecer la importancia de asumir posturas pedagógicas claras en pro de formar una ciudadanía que incluya la diversidad, la tolerancia, la alteridad, el respeto absoluto por el otro, la inclusión de todos los integrantes para la construcción de un proyecto común.

Este proyecto parte de la apuesta de Giroux del papel de los profesores como agentes "recuperadores" de su dimensión cívica, reconstruyendo un lenguaje visionario y una filosofía pública que pongan en igualdad la libertad y la vida humana en el centro de los conceptos de democracia y ciudadanía (1988, p. 222), lo que implica una apuesta por reformar tanto el currículo de las facultades de formación de formadores como la más difícil toma de conciencia de aportar por unos procesos pedagógicos que incluyan el aula, la calle y el aura de un mundo para mundializar y no globalizar.

En su postura conceptual se marcaron tendencias como liberalismo, comunitarismo y republicanismo, definiendo que la ciudadanía no es sólo conocimientos, sino una acción social y política de los individuos (p. 26), y que, en el proceso educativo para la ciudadanía, los dispositivos didácticos no se circunscriben sólo a las aulas, sino a la integración de los espacios y actividades académicas de la institución. Además, se deben vincular los procesos cognitivos, comunicativos, emocionales, actitudinales, de acción en conjunto con la imaginación, la voluntad y el deseo hacia lo político y social, para que los individuos entiendan su ser sujetos políticos y sean responsables de sí mismos y de los demás.

Se establece que el aprendizaje basado en problemas (ABP) es un método expedito para desarrollar estos procesos de formación en ciudadanía, porque permite la negociación cultural que implica convivencia de saberes, estilos de aprendizaje, sentidos y percepciones diversas. Así se forma al ciudadano para ser agentes de paz, lo que favorece la relación ciudadanía, posconflicto, reconciliación, justicia y paz. 
La construcción de ciudadanía es una tarea multidiversa, que implica la integración de todas las estancias sociales en función de lograr una nación que ha sido afectada por la violencia institucionalizada. Desde la educación, se intenta que en el aula se generen estudiantes y maestros pedagogos (no es un oxímoron esta relación) como sujetos políticos que transformen su entorno vital. Es así que Fernández y Echevarría (1998) se preocuparon por mostrar cómo la búsqueda de una mejor calidad de vida y la construcción de sociedades más democráticas en lo local, regional y nacional, son parte del proceso pedagógico de constitución de la ciudadanía, y cómo esta construcción está inmersa en la tensión cultural entre el cruce de paradigmas para una ciudad golpeada por la violencia, como es el caso de Medellín. En su trabajo "Educación y ciudadanía, notas desde la experiencia de Medellín", se preguntaron ¿Cómo la educación ciudadana aporta al fortalecimiento de las nuevas formas de comprensión de la política, la economía y la vida social influenciados por procesos globalizantes y culturales que llevan a concebir la realidad de modo interdisciplinar, multicultural y multiétnico? (p. 32). Comprendieron que todo proceso pedagógico de formación en ciudadanía está íntimamente ligado a los procesos políticos y culturales del entorno, y que debe estar en consonancia con la perspectiva de ciudadanía del mundo que piensa global y actúa local.

Partiendo de esta visión, concluyeron que la ciudadanía es un estilo de vida que se conquista, se construye en la medida en que el individuo se inserta en una comunidad que lo acepta en la totalidad de su dimensionalidad, se compromete en los proyectos comunes a que llegaron mediante el disenso y consenso, y basados en el respeto y la tolerancia, intentan solucionar los problemas que aquejan al entorno inmediato, desde una mirada global, no globalizada, es decir, bajo un pensamiento glocal (Cortina, 2000, p. 220ss). Así, la ciudad, como escenario público de construcción de imaginarios, ideologías y proyectos, se convierte en educadora que busca una vida digna para todos sus miembros, haciendo realidad lo que Cortina habla de un Estado de Justicia (2010).

Ahora bien, en la formación de ciudadanos se hace evidente la vinculación del tema trasnacional, en cuanto que el sujeto se está comprendiendo como un ciudadano del mundo que hace parte activa de la construcción de una sociedad trasnacional, con diferentes instituciones y organizaciones, cuya finalidad es el acercamiento del mundo a cada sujeto (Montoya, 2011, p.35). La ciudadanía también 
comprende un ejercicio de identidad en medio de una sociedad cambiante por los diversos procesos culturales, demográficos, políticos, sociales y económicos, como fue el caso de la planeación del desarrollo y las veedurías ciudadanas que han dado resultado, a la hora de generar espacios de participación ciudadana, lo cual ha sido producto de la aplicación de procesos educativos y pedagógicos en ciudadanía, lo que implica que no puede seguirse basándose en conceptos estáticos y cerrados que redundan en nacionalismos que, llevados al extremo, pueden generar sistemas opresores, violentos y transgresores de la misma humanidad.

\section{Ciudadanos formados para el mundo}

Unido a estos problemas que se han venido desarrollando, a lo largo de este trabajo, en la actualidad es vital integral el tema de la migración a la hora de comprender la ciudadanía en el sentido de ser ciudadanos del mundo, y así mismo, el papel de la educación y la pedagogía crítica. Por eso Muñoz (2005) afirmaba que los procesos migratorios han posibilitado una resignificación del término ciudadano en relación con la posición de éste frente a su situación política mundial, y que debe ser considerado como un sujeto en una aldea global donde la tecnología, los alcances del poder de la información, y la intercomunicación de idiomas, costumbres e idiosincrasias que plantea McLuhan (2005). Y la pedagogía debe generar procesos de participación en medio de las aulas para que asuman su papel político en medio de las sociedades, no en una cuestión de libros como lo afirmaría Castoriadis (1998, p. 123).

Es una condición que se conciban como sujetos libres, crítico y reflexivos, conscientes de sus derechos, capaces de poner en juego sus intereses y sus demandas y dispuestos a ejercer influencia en las decisiones públicas (p. 78). En este horizonte, el docente debe ser ciudadano que pone su labor al servicio de la persona y no de los poderes fácticos de turno. Así pues, la ciudadanía democrática ejercida por el docente debe superar por un lado la "involución política del ciudadano" (Saavedra, 2002), es decir, recuperar sus derechos negados por el neoliberalismo y superar la crisis de ciudadanía negada en las aulas contemporáneas. Llega a una conclusión importante, citando a Giroux, en que el docente debe ser un intelectual público que afronte el mundo abordando los problemas con seriedad. Así la cuestión del aprendizaje 
se vincula con formas de activismo que aumentan la posibilidad de una vida democrática (1994).

Por tanto, se ha podido describir los diversos trabajos realizados acerca de la relación entre pedagogía y ciudadanía, encontrándose que no aparece este binomio, como sí aparecen la ciudadanía unida a categorías como formación, educación, integración. En la presentación de las diversas pedagogías, algunas la presentan como una meta a la que se podrá llegar, adjunta a otras metas como integralidad y humanización del entorno social. Además, se evidencia la importancia, tanto de la comprensión como de la misma formación en ciudadanía en la escuela, aunque no se hace evidente la íntima relación existente entre la práctica pedagógica y la ciudadanía, lo que será nuestro aporte desde nuestra investigación, bajo la perspectiva de la pedagogía crítica y la lente conceptual de Adela Cortina sobre ser ciudadanos de y para el mundo. Se destaca la importancia del tema en las diversas ciencias sociales y humanas, dada su condición de ser una categoría integradora e interdisciplinar, y debido a sus implicaciones políticas, culturales y económicas (Giraldo-Zuluaga, 2015, p. 3).

Se reconoce la importancia de la experiencia de la vida cotidiana para lograr una formación en ciudadanía, lo que vincula academia y cotidianidad en las prácticas pedagógicas, de tal manera que cada estudiante podrá problematizar sobre sus propias vivencias, buscarán en comunidad soluciones prácticas a las que se podrán comprometer, entendiendo así que podrán formar parte activa de los proyectos de la sociedad, dejando de lado la condición de ciudadanos pasivos, y generar un pensamiento autónomo, creativo y crítico ante los problemas tanto de orden local como de orden global.

En la formación en ciudadanía es necesario tener presentes temas como el cuidado del medio ambiente, dar oportunidades para la toma de decisiones, tomar parte de una acción prosocial, forjar habilidades en resolución de conflictos y generar espacios en que se pueda hacer frente a la injusticia (Cárcamo-Vásquez, 2015, p. 3). Para que esto sea una realidad, los futuros docentes deben, en sus primeras fases formativas, reflexionar y trabajar críticamente en estos aspectos para que sus próximos estudiantes comprendan los diferentes temas bajo estas premisas que sí forman integralmente a la persona, desde una perspectiva habermasiana en cuanto que lo fáctico es fruto histórico de intervenciones, creencias, discursos y relatos que permiten una emancipación ante los sistemas 
hegemónicos y totalitarios que alienan la condición humana en su propio mundo de la vida (Salvat, 2014, p. 96). Unido a ello, y en unas coordenadas de multiculturalismo e interdisciplinariedad, es vital que cada agente del acto pedagógico asuma nuevas posibilidades políticas, incluso en el aula, de acceso a la diferencia y a la experiencia de la tolerancia con los diversos movimientos de diversas naturalezas que promueven la construcción de nuevas subjetividades e identidades dialógicas con el otro y lo otro (Hernández., 2009, p. 129), basados en el respeto al que es diferente, el cual es un sujeto en igualdad de condiciones y posibilidades, y que en el encuentro con éste se genera una lectura polifónica, es decir, a diversas voces, discursos, relatos y memorias. Esto permitirá cuestionar y eliminar los prejuicios para posibilitar la construcción de nuevos contextos que favorezcan la participación ciudadana (Fajardo, 2016, p.96)

Cárcamo-Vásquez plantea que las representaciones sociales son vitales para la formación en ciudadanía, pues son un "corpus organizado de conocimientos y una de las actividades psíquicas gracias a las cuales los hombres hacen inteligible la realidad física y social" (Moscovici, 1979, citado por Cárcamo-Vásquez, 2015, p. 17), pues son construidas por la comunidad específica que decodifican sistemas de creencias, valores y estereotipos que orientan el actuar de los sujetos y las formas de relacionarse con el mundo. Así la pedagogía juega un papel preponderante en vista que integra saberes tanto específicos como de las formas de llegar a concretar nuevas ideas en las mentalidades de los estudiantes.

Cárcamo sostiene que en la formación en ciudadanía se pone el acento en la práctica específica referida al método de enseñanza que el profesor puede o debe utilizar, que la ciudadanía se forma desde el aula, de tal manera que los estudiantes aprendan y se sientan incorporados a la sociedad. Estas son sus palabras:

La representación de "persona educada" unida a la idea de educación sustentada en la estandarización, el orden y la reproducción cultural que se aprecia entre quienes estudian para ser maestros y maestras de primaria, se ve reflejada en las concepciones que poseen sobre la ciudadanía (en la que prima la perspectiva política) y en las formas sociales que consideran apropiadas para promoverla (el orden y el respeto), en tanto que competencia social y ciudadana al interior del espacio escolar (Cárcamo-Vásquez,2015, p. 11). 
Por tal razón se puede comprender la distinción que Giraldo y Zuluaga hacen de ciudadanía entre actividad y condición. La primera es heredera de la historia y pensamiento político que la asumen como "estilo de vida" (2015, p. 5), visión ésta que data desde la ilustración en la que los individuos hacen parte de la sociedad si son educados para ser ciudadanos, y en la medida de sus posibilidades (entiéndase capacidades) participan en la vida política. En el caso de la segunda acepción, el individuo tiene la condición de ciudadano, gracias a las revoluciones del siglo XVII y el nacimiento de las Repúblicas, en cuanto que nacen como ciudadanos, hijos de los Estados Nación basados en principios liberales y cobijados por la ley que los cataloga como verdaderos ciudadanos que construyen "verdades" para liberar las mentalidades con autocrítica y evitar volver al error (Morin, 2015, p.18).

\section{Concluyendo: la ciudadanía como don y tarea}

La condición de ser ciudadano comporta la actividad, en cuanto que adhesión y reconocimiento, en palabras de Cortina, "son las dos caras de la moneda que, al menos como pretensión, componen ese concepto de ciudadanía que constituye la razón de ser de la civilidad" (Cortina, 1998, p.25). Desde este sentido, podría parafrasearse que es un don, no de orden divino, sino social que se adquiere mediante un proceso formativo y dialógico entre los diferentes actores que se asumen como individuos autónomos y codependientes con el otro y lo otro, en el que se integra la idea del bien común, de la libertad, de la construcción de sujetos y verdades en contextos de nuevas ideas culturales y perspectivas universalistas. Así lo expresan García y Muñoz cuando se asiste a la entrada a una nueva era del conocimiento y la información que deben cambiar nuevas pautas de desarrollo y construcción de identidades comunitarias y personales, las cuales se convierten en un nuevo contexto para lo que definieron como pedagogía de los espacios (2004, p. 257) y en estos la ciudadanía se convierte en un nuevo lugar pedagógico para todo maestro y pedagogo que se autocomprende como un sujeto político en palabras de Freire (1990).

Ahora bien, el concepto de Marshall (1998) configuró a la ciudadanía su status de condición o estado de todos los individuos, quienes, además 
de poseer de suyo el derecho a una comunidad en igualdad, ésta debía tener también el acceso real al disfrute de esos derechos y garantías sociales, sociales, económicas y culturales. En este contexto nacieron los estados de bienestar, los cuales, según Giraldo y Zuluaga, permitieron que se hiciera posible la universalización de los derechos socioeconómicos y se incorporaran al ciudadano $(2015$, p. 7). Así, esta visión que se ha denominado, a partir de este autor, como "clásica", estableció que se integraran tres características o tipologías de la ciudadanía: civil que comprende los derechos y las libertades individuales, política que configura los derechos políticos, y una social que abarca los derechos socioeconómicos, sociales y culturales (OEA-PNUD, 2009).

Bedoya y Santana comprenden la ciudadanía como "la inserción y expresión participativa y perteneciente del individuo humano en los espacios públicos, con derechos, deberes y accesos comunitarios, civiles, políticos, sociales, económicos y culturales con el objeto de hacer realidad la democracia participativa" (2003, p. 2). A partir de esta definición se denota la necesidad de resignificar el papel del ciudadano en medio de su posición ante el proyecto común de la sociedad, a partir de lo que Hoyos comentaba de racionalidad comunicativa, la cual se convierte en tarea en cuanto darse a la tarea de vigorizar el mundo de la vida (2007, p. 3; 1990, p. 32) y fortalecer la opinión pública que se convierte en el escenario para formar sujetos activos, racionales, de palabra y capaces de llegar a acuerdos. Este es uno de los aspectos donde la ciudadanía es una tarea.

La vivencia de la democracia se evidencia mediante la participación activa de los ciudadanos que desarrollan sus diversas dimensiones como su libertad, el desarrollo humano (sostenible), la igualdad social, política y moral, los cuales son derechos instituidos y que deben ser garantizados por el Estado, a lo que Cortina establecería como la expresión válida de un Estado de Justicia (2012), de tal manera que la ciudadanía y la democracia van más allá de lo político, pues abarca las dimensiones, necesidades y espacios humanos donde actúa el ser humano, aunque ha de hacerlo de manera crítica en orden a la emancipación y análisis responsable de las dinámicas sociales que intentan ejercer un control económicos y social de la vida del ser humano, de tal forma que éste se convierta en un simple "reproductor social y cultural de una sociedad marcada por desigualdades significativas de poder, privilegio y riqueza" (Giroux, 2004, p. 216), a lo que Cortina establecerá como conditio sine qua non la autorreflexión que fundamente la realidad dialógica del sujeto determina su conducta a partir de los consensos a los que llegó con su comunidad que aceptó y 
comprometió con la normatividad (1985, p. 158) que se impone porque la asumen como su deber ser.

Para la formación en ciudadanía se encuentra la necesidad de vincular al estudiante en los proyectos comunes, rechazar la tendencia a buscar las certezas absolutas y resignificar la realidad desde diversos contextos, ya que solo así el aprendizaje es contextualizado, significativo y comprometido con las experiencias mismas de los estudiantes. De esta forma lo demuestran Lúquez et al. (2012), quienes realizaron, mediante una metodología de aprendizaje por servicio, unos ejercicios de participación y protagonismo de los estudiantes en un contexto de diversidad cultural, favoreciendo la responsabilidad social de los universitarios. Este estudio realizado por los autores permitió inferir la imperiosa necesidad de resignificar y reestructurar el currículo de la formación en ciudadanía en relación con las demás cátedras tanto en la educación básica como la superior, y más aún en la emergente terciaria y continuada, donde se vinculen las emociones, las competencias cognitivas y las ético-sociales (Luquez et al., 2012, p. 482) de cara a la configuración de un mundo más humano que respete incluso lo vivo no humano.

Así, el estudiante aprende a tomar control de sí mismo y en compañía del otro, toma posesión de los destinos de su entorno. Para formar la paz es vital tener en cuenta algunos criterios: aprender para la individuación, aprender de lo diferente, aprender para la acción transformadora, aprender a aprender, aprender del conflicto, aprender a cambiar en medio del cambio y aprender a empoderarse produciendo conocimiento (Bedoya-Santana, 2003, p. 3). De manera indiscutible una de las tareas de la ciudadanía como tarea, esto es un proyecto inacabado ha de ser la lucha por no seguir en el discurso controlador de preparar consumidores en lugar de ciudadanos, partiendo de la ineludible e inextricable primacía de los valores democráticos sobre la cultura empresarial y los valores comerciales (Giroux, 2003, p. 83) en un contexto relacional entre el ser humano y todo lo vivo no humano. Al respecto, Cortina plantea que es necesario definir un ethos, es decir, "un carácter personal y social predispuesto a no expoliar la naturaleza, sino a colaborar en su desarrollo" (2012, p. 145), porque no basta con buscar soluciones tecnológicas a un gran problema que tenemos delante nuestro, es decir, con la tecnología se solucionan algunas problemáticas, generando otras que pueden ser más graves aún. De ahí que una respuesta preventiva eficaz sea el cultivo de una actitud, entre 
todas las personas, ante la naturaleza sin manipularla y hacerlo público ante los demás.

El ciudadano ideal es una tarea de todos los maestros, y la pretensión de este trabajo lo buscará mediante un proceso pedagógico de apropiación del entorno, la generación de espacios democráticos de tal forma que sea aquel cuyas actitudes y comportamientos se ajusten a los valores relativos a la interacción democrática (libertad, igualdad jurídica, pluralidad, tolerancia, respeto, diálogo, negociación, pluralidad y participación), al cumplimiento de las obligaciones sociales (responsabilidad familiar, escolar, laboral, etcétera), a la autorrealización (sujeto autónomo), a la ayuda al más débil (solidaridad) y a la defensa de un medioambiente saludable y sostenible (Citado por Giraldo \& Zuluaga, 2015, p. 14). Unido a esto, un nuevo contexto que debe asumir la educación y la pedagogía es la búsqueda de escenarios para la democracia y la ciudadanía global, a partir de conocimientos que integren la vida, la justicia y la transformación del mundo de la vida (Cortina, 1995, p. 41). En este mismo orden de ideas es importante mencionar unas palabras de Adela Cortina en una conferencia que impartió en el año 2009:

El tercer pilar de una democracia comunicativa son sus
ciudadanos. Y aquí, como en los demás puntos, vuelve a ser
central el papel de la educación porque es necesario formar
ciudadanos autónomos y solidarios, capaces de formarse
un juicio sobre lo justo, preocupados por descubrir junto con
sus conciudadanos qué es lo justo, empeñados en la tarea de
ponerlo por obra en la vida cotidiana. Para eso hace falta -creo
yo-cultivar una razón cordial. Porque conocemos la justicia no
sólo por la razón, sino también por el corazón. (2009, p. 26)

Por estas razones, este ciudadano debe ser visto, como se ha intentado sostener en este texto, como un sujeto que responde a las necesidades no solamente de su mundo de la vida inmediato, sino que comprende su posición y acción en relación con el mundo en donde supera la concepción clásica y se abre a la multiculturalidad (Garzón, 2013, p. 76) y al cosmopolitismo que permite vislumbrar la utopía de una humanidad común universal que supera los límites dados por las políticas hegemónicas del sistema capitalista, a lo que Tejedor y Rodríguez (2008) complementan que una de las labores de los docentes, maestros y pedagogos ha de ser el reordenamiento del acceso al conocimiento y su discernimiento para crear escenarios transformadores de la condición 
humana con acciones significativas que elimine toda tendencia a la desigualdad social $y$, en palabras de Giroux, asuma un papel de pie de lucha por reivindicar el papel de la universidad ante los dispositivos de control tanto nacional como a nivel internacional (2003, p. 42) que socavan y dejan en el desierto poblado de aullidos las iniciativas emergentes de la academia universitaria por transformar el mundo humanizando al hombre que está advirtiendo un nuevo mundo en el que sólo será posible experimentarlo en un diálogo y pensamiento desde el Sur, es decir, lo que Hoyos (2011) comenta de iniciar el proceso crítico de liberarse de las lógicas del mercado y pensar el mundo en un horizonte de comprensión de apertura al mundo con valores que se complementan y traducen en comportamientos humanizados de respeto al otro y lo otro.

\section{Referencias bibliográficas}

Bogoya, N. (2003). Hacia una pedagogía para la ciudadanía. Infancias. 5(1), 24-27.

Cabezudo, A. (2006). Ciudad Educadora, una manera de vivir juntos. Disponible en: http://www.hegoa.ehu.es/congreso/gasteiz/doku/Ciueduc.pdf

Camps, V. (2010). Democracia sin ciudadanos. La construcción de la ciudadanía en las democracias liberales. Madrid: Trotta.

Cárcamo-Vásquez, H. (2015). Ciudadanía y formación ciudadana: ¿Qué ocurre en el aula donde se forma el futuro profesorado de educación primaria de una universidad pública de Madrid? Revista Electrónica Educare, 19(3), 1-14. doi: http://dx.doi.

org/10.15359/ree.19-3.3

Castoriadis, C. (1998). Los dominios del hombre: las encrucijadas del laberinto. 1a edición. Barcelona: Gedisa.

Cortina, A. (1998). Ciudadanos del mundo, hacia una teoría de la ciudadanía. Madrid: Alianza.

Cortina, A. (2000). Ética Mínima. Introducción a la filosofía práctica. 6ạ. Edición. Madrid: Tecnos.

Cortina, A. (2000). Ética sin moral. 4ạ. Edición. Madrid: Editorial Tecnos.

Cortina, A. (2003). La inocencia robada. Juventud, multinacionales y política cultural. Madrid: Ediciones Morata. 
Cortina, A. (2009). Las raíces éticas de la democracia. Conferencia en la Universitat d’Estiu de Gandia. Valencia: Universidad de Valencia.

Cortina, A. (2010). Justicia cordial. Madrid: Trotta.

Cortina, A. (2004). Por una ética del consumo. La ciudadanía del consumidor en un mundo global. Madrid: Taurus.

Cortina, A. (1985). Razón comunitaria y responsabilidad solidaria. Salamanca:

Ediciones Sígueme.

Cortina, A. (1993). Ética aplicada y democracia radical. Editorial Madrid: Tecnos.

Cortina, A. (1995). La educación del hombre y del ciudadano. En: Revista

Interamericana de Educación. Nro. 7, enero-abril. Disponible en: rieoei.org/oeivirt/ rie07a02.pdf

Fajardo, P. (2016). Hacia la caracterización de los valores democráticos y ciudadanos de los estudiantes universitarios: una mirada desde la formación política y la construcción de paz. En: Revista Interamericana de educación, pedagogía y estudios culturales. Volumen 9. Número 1. Enero - Junio 2016 USTA, Bogotá.

Fernández, R. Echeverría, M.C. (1998). Educación y ciudadanía, notas desde la experiencia de Medellín. Nómadas, 9, 130-140.

Freire, P. (1990). La naturaleza política de la educación. Cultura, poder y liberación. Barcelona: Paidós.

García del Lujo, A. Múñoz, J.M. (2004). Pedagogía de los espacios. Esbozo de un horizonte educativo para el siglo XXI. Revista Española de Pedagogía. Año LXII, 228, 257-278.

Garzón, P. (2013). Sobre la indeterminación conceptual de la ciudadanía multicultural. El Cotidiano. 180, 75-88.

Giraldo-Zuluaga, G.A. (2015). Ciudadanía: aprendizaje de una forma de vida. Revista Educare. Vol. 18(1), 76-92. DOI: 10.5294/edu.2015.18.1.5

Giroux, H. (1994). Pedagogías itinerantes, placeres inquietantes. Buenos Aires: Paidós.

Giroux, H (1988). Los profesores como intelectuales. Hacia una pedagogía crítica del aprendizaje. Paidós, Barcelona.

Giroux, H (2003). La inocencia robada. Juventud, multinacionales y política cultural. Ediciones Morata, Madrid

Giroux, H (2004). Teoría y resistencia en educación. Una pedagogía para la oposición. Siglo XXI editores, México. 
Giroux, H (2008). La Universidad secuestrada. El reto de confrontar a la alianza militarindustrial-académica. Caracas: Ministerio del Poder Popular para la Educación Superior.

Hernández Acevedo, A. (2015). El sujeto hi-stórico, un reto para el maestro pedagogo. Revista Espirales. Vol. 3(3), 8-17.

Hernández Acevedo, A (2016). Apuntes de clase de pedagogía crítica.

Hernández, K. (2009). Nociones básicas sobre multiculturalismo y sus interacciones con los derechos humanos. Derechos humanos México, Año 4 (11). Disponible en: https:// revistas-colaboracion.juridicas.unam.mx/index.php/derechos-humanos-cndh/article/ view $/ 5673 / 5010$

Hoyos, G. (1990). Prólogo a: Díaz, M. Muñoz, J.A. Pedagogía, discurso y poder. Bogotá: Corprodic.

Hoyos, G. (2007). Comunicación, educación y ciudadanía. Conferencia inaugural. Maestría en educación 21 de agosto. Cali: Universidad del Valle. Instituto de Educación y Pedagogía.

Hoyos, G. (2011). Educación y ética para una ciudadanía cosmopolita. En: Revista Iberoamericana de Educación. 55, 191-203.

Kymlicka, W. (1996). Ciudadanía multicultural: una autoría liberal de los derechos de las minorías. Barcelona: Paidós.

Luquez P,, Fernández, O., Sanservero, I., Fontanilla, N. (2012). Praxis de compentencias ciudadanas en el ejercicio de la Responsabilidad Social Universitaria. Opción, Año 28(69), 480-497.

Magendzo Kolstrein, A. (2007). Formación de estudiantes deliberantes para una democracia deliberativa. Revista Electrónica Iberoamericana sobre Calidad, Eficacia y Cambio en Educación. REICE. 5(4), 70-82.

Marshall, T.H. (1998). Ciudadanía y clase social. Madrid: Alianza editorial.

McLuhan, M., Powers, B.R. (2005). La aldea global. Transformaciones en la vida y los medios de comunicación mundiales en el siglo XXI. Barcelona: Editorial Gedisa.

Ministerio de Educación Nacional. (2006). Estándares básicos de Competencias ciudadanas. Bogotá: MEN.

Ministerio de Educación Nacional. (2013). Módulo de Competencias Ciudadanas. Saber Pro. Bogotá: ICFES.

Montoya, L.(2011). Educación, derechos humanos, política y ciudadanía. Documento de fundamentación de una línea de investigación del Doctorado en Educación de la Universidad Santo Tomás. En: Revista Interamericana de educación, pedagogía y estudios culturales. Vol. 4 - No. 1 / Bogotá, D.C. - enero - junio 2011 / pp. 33 - 52. 
Moreno, ND., Chilito, O.E., Trujillo, J.O. (2010). Juventud y ciudadanía desde la educación en la calle. Revista Colombiana de Ciencias Sociales. 1(1), 2-27.

Morin, E. (2015). Enseñar a vivir. Manifiesto para cambiar la educación. Buenos Aires: Ediciones Nueva Visión.

Muñoz, W. (2005). La ciudadanía pedagógica, una reflexión de ciudadanía democrática desde el proceso educativo. Revista Horizontes Educacionales. 10(1), 77-83.

OEA-PNUD. (2009). La democracia de ciudadanía. Una agenda para la construcción de ciudadanía en América Latina. Disponible en: http://www.oas.org/dsp/documentos/ Publicaciones/La_democracia_de_ciudadania.pdf

Paz, M.A., Venturino, C. (coords.). (2008). Introducción al diseño curricular: construcción ciudadana. 1a. Edición. Buenos Aires: Dirección General de Cultura Educación de la Provincia de Buenos Aires.

El Tiempo. (1997). Proyecto ciudadano en Tunja. Disponible en: http://www.eltiempo. com/archivo/documento/MAM-673600

Ramírez Saíz, J.M. (2012). Dimensiones Constitutivas y ejes estructurales de la ciudadanía. Estudios Políticos. 9(26), 11-36.

Saavedra, S. (2002). Discusiones en torno al concepto de ciudadanía. En: revista SER, Servicio de Estudios Regionales. Nro. 1, Vol. 29, Julio, Santiago de Chile

Salvat, P. (2014). Habermas: la democracia deliberativa como democracia radical. En: Figueroa, M. (editor). Poder y Ciudadanía: estudios sobre Hobbes, Foucault, Habermas y Arendt. Santiago de Chile: RIL editores.

Streck, D.; Zanini Moretti, C. (2013). Colonialidade e insurgência: contribuições para uma pedagogia latino-americana. Revista Lusófona de Educação, 24, 35-52.

Tejedor, M., Rodríguez, H. (2008). Ciudadanía y aprendizaje dialógico en los programas universitarios para mayores: reflexiones y experiencias desde la pedagogía crítica. Educar. 42 97-115.

Valencia, G.C. \& Giraldo, G. (2005). La formación de ciudadanos: proceso tensional entre las culturas escolar y no escolar. Documento de trabajo versión preliminar. Cali: Universidad San Buenaventura. 